\title{
Allosteric effect by synchronized resonance of amide bonds through alpha-helix
}

\author{
Hiroyuki Aizawa* \\ Aizawa Science Museum, Kasukabe, Saitama, Japan
}

\begin{abstract}
Alpha-helix is one of the major second structures of polypeptides. Alpha-helix is stabilized by hydrogen bonds between carbonyl residue of amino acid at position $\mathrm{N}^{\text {th }}$ and amine residue of amino acid at position $\mathrm{N}+4^{\text {th }}$. Each amide bond could take either one of keto-type and enol-type while the former has lower Gibb's free energy than the latter. Resonance between the two types makes the amino bond planar to stabilize alpha-helix. Although static structure of alpha-helix is well studied by X-ray crystallography, its dynamic nature is not fully understood yet. Here, we propose one model of dynamic behavior of alpha-helix in solution. Without synchronized resonant reactions, all amide bonds in alpha-helix prefer keto-type to enol-type losing their planar structure. Binding to amide bonds at the end of alpha-helix, hydrogen donor and acceptor group such as carboxyl group and phosphate group could induce synchronized resonance of amide bonds between ketotype and enol-type at high frequency. Especially when polypeptides form anti-parallel alpha-helices with two ligands binding at both ends, they make a closed chain reaction of synchronized resonance at maximal frequency. Accordingly, one ligand binding at an end caused high affinity binding of another ligand on the other end of the antiparallel alpha-helices. This model suggests a fundamental molecular mechanism of an allosteric effect of protein complex, through which alpha-helices transfer chemical binding signals from one site to another by synchronized resonance reactions.
\end{abstract}

\section{Basic structure of alpha-helix}

As Dr. Pauling suggested, alpha-helix consists of right-handed helix of a polypeptide, which is stabilized by hydrogen bonds among amide residues $[1,2]$. In an alpha-helix, all the carbonyl oxygens of amide bond extend forwardly toward the axis of the helix, while all the amine hydrogens of amide bond extend backwardly along the axis of the helix. Each carbonyl oxygen of amino acid residue at position $\mathrm{N}^{\text {th }}$ makes a hydrogen bond with an amine hydrogen at position $(\mathrm{N}+4)^{\text {th }}$ to stabilize alpha-helix structure (Figure 1). All the side chains of amino acid residues extend laterally toward outside of the helix. This is a unique nature of alpha-helix when compared to beta-sheet where neighboring side chain residues locate on the opposite side of the sheet to each other.

In the lipid bilayer, transmembrane alpha-helix should extend hydrophobic groups of side chain outside to interact with hydrophobic alkyl chains of fatty acids for stabilization [1,2]. Since there are no polar groups within the hydrophobic layer of lipid bilayer, amides of a transmembrane polypeptide readily form hydrogen bonds among them $[1,2]$. Thus, alpha-helix is one of stable structures of transmembrane polypeptides in the lipid bilayer.

In an aqueous solution, alpha-helix should extend hydrophilic groups of side chains outside to interact with hydrophilic water molecules and salt ions in a solvent for solubilization [1,2]. Water molecules are so polar that hydrogen bonds among amide bonds within alpha-helix are easily attacked by water molecules to be broken while non-polar alpha-carbons tend to locate at the center of the alpha-helix with hydrophobic interactions. Generally, alpha-helix in an aqueous solution is less stable than that in a lipid bilayer. Inside of the protein complex, however, an alpha-helix may be much stable in a hydrophobic environment just like that in a lipid bilayer. In such a case, alpha-helix could be stabilized by stable hydrogen bonds among amide bonds which are surrounded by hydrophobic side chains.
A

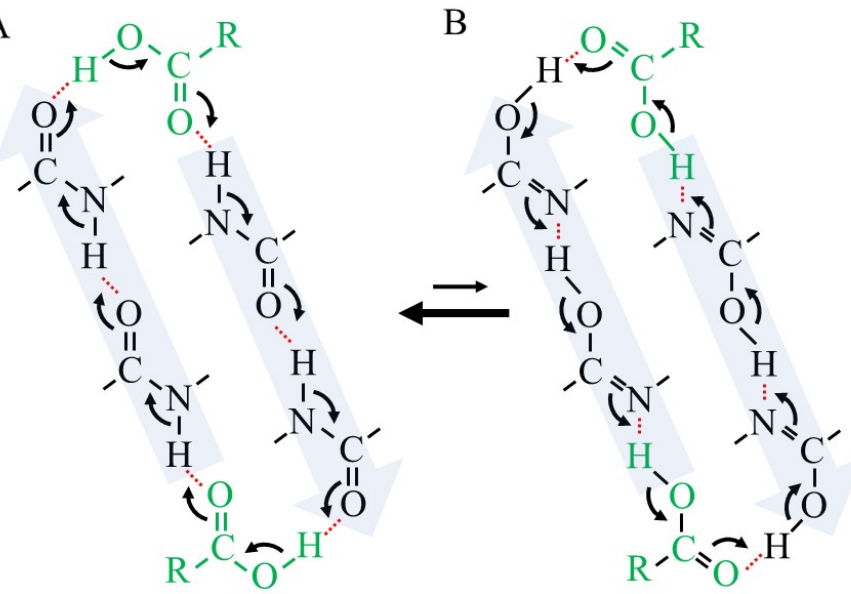

Figure 1. Schematic model of an allosteric effect of carboxyl group binding by a closedcircular resonant reaction in a pair of anti-parallel alpha-helices. A, keto-type structure of alpha-helix. B, enol-type structure of alpha-helix. In this schematic cartoon, a carboxyl compound, which binds to one end of anti-parallel alpha-helices, stimulates another carboxyl compound to bind to the helices on the other end by resonant stabilization. Only three residues of each alpha-helix were presented for simplicity. A black solid line indicates a covalent bond. A red dotted line indicates a hydrogen bond. A curved arrow indicates electron transfer or negative electric flow during a resonant reaction. A pair of anti-parallel black allows indicates chemical equilibrium between keto-type and enol-type. A green $\mathrm{R}-\mathrm{COOH}$, a carboxyl compound

*Correspondence to: Hiroyuki Aizawa, Aizawa Science Museum, 1-10-13 Yutaka-cho, Kasukabe, Saitama 3440066, Japan, Tel: +81-48-754-9880; Fax: +8148-754-9880, E-mail: aizawa@rr.iij4u.or.jp

Key words: allosteric effect, alpha-helix, dynamics, hydrogen bond, amide resonance

Received: June 28, 2018; Accepted: July 09, 2018; Published: July 11, 2018 


\section{Planar structure of amide bonds in a peptide}

In a short peptide, amide bonds always take a planar structure by resonance between keto-type and enol-type in an electrolytic solution. Amine and carbonyl residues work as hydrogen donor and acceptor in solution, respectively. Once the amine hydrogen is released to and accepted by some solvent molecule, hydrogen transfer chain reaction occurs in solution until the carbonyl oxygen accept hydrogen atom from a solvent molecule, resulting in transformation of the amide residues from keto-type to enol-type. Because keto-type has lower Gibb's free energy than enol-type, all the enol-type amides should quickly return to keto-type reversibly. This resonance reaction forms planar structure of amino bonds with resonant formation of double bonds among oxygen, carbon, and nitrogen [3]. The frequency of resonance depends on the electric resistance between the donor and acceptor and thus should be positively corelated to the ionic conductivity of the solution.

Within alpha-helix, however, all the amide bonds make a chain of hydrogen bonds to one another without direct interaction with solvent. To synchronize all the resonant reactions of amide bonds of an alphahelix, a carboxyl and amine ends of the helix should accept hydrogen from and donate hydrogen to the solvent, respectively. Since alpha-helix takes a long helical structure, electric resistance between both ends of the helix is much higher than that of a short peptide for donation and acceptance of hydrogen in the solution. Accordingly, frequency of synchronized resonance of amide bonds in alpha-helix is much lower than that of a short peptide in solution. Consequently, planar structure of amide bonds is less stable in an alpha-helix than in a short peptide, especially in a solution with low ionic strength.

\section{Dynamics of alpha-helix}

Thermodynamically, keto-form of an amide bond is more stable than its isoform or enol-type [1,2]. Thus, almost all the amide bonds prefer keto-type to enol-type as mentioned above. Moreover, both carbonyl and secondary amine residues are so weak electrolytes in solution that amide bonds in alpha-helix performs synchronized resonance at low frequency. However, once a strongly electrolytic group such as carboxy group and phosphate group associates with the amide bond at the end of alpha-helix, all the amide bonds of the helix become resonant synchronously between keto- and enol-form at high frequency. In other words, strong electrolytes increase conductivity of the alpha-helix in solution. The resonance at high frequency stabilizes planar structure of each amide bonds, resulting in stabilization of alpha-helical structure.
Since the resonant reactions synchronized to one another all through the helix, the dynamic stabilization of alpha-helical structure occurs not only locally at the end but also through the entire helix.

\section{Allosteric mechanism of alpha-helices}

As shown in Figure 1, anti-parallel alpha-helices could transfer molecular information of ligand binding from one site to another within a protein as an electric cable communication system. In other words, ligand binding at one end stabilizes ligand/substrate binding at another end of the alpha-helices by synchronized resonant reactions, which is well known as an allosteric effect [4,5]. In this model, a ligand could have hydrogen bonding residues especially such as a carboxyl group and a phosphate group to couple the resonant reactions. Furthermore, allosteric effect accompanies with stabilization of planar structure of amide bonds all through the helices as discussed above.

It is also possible that the allosteric mechanism plays a central role in the signal transduction of transmembrane receptors or channels between outside and inside of lipid bilayer through their alpha-helical transmembrane peptides. For example, ligand binding at an outside domain of a transmembrane receptor could transform structure and dynamics of the transmembrane alpha-helices, which induces substrate to its intracellular domain [1].

It should be noted that all the models proposed here are just a theoretical hypothesis from the nature of hydrogen bonding between amide bonds of polypeptide, and thus should be carefully examined by experiments in the future. It should also be confirmed in silico that the model proposed here should be applicable with acceptable accuracy to published atomic structures of proteins which are known to have allosteric effects.

\section{Conflicts of interest}

The author declares no conflict of interest.

\section{References}

1. Cox MM, Nelson DL (2012) Principles of Biochemistry, W.H. Freeman; $6^{\text {th }}$ edition.

2. Pauling L (1988) General Chemistry, Dover Publications; $3^{\text {rd }}$ edition.

3. Aizawa H (2018) On the structure of beta-sheet. J Mol Cell Biol Forecast 1: 1003.

4. Motlagh HN, Wrabl JO, Li J, and Hilser VJ (2014) The ensemble nature of allostery. Nature 508: 331-339.

5. Liu J, Nussinov R (2016) Allostery: An overview of its history, concepts, methods, and applications. PLOS Comput. Biol 12: e1004966.

Copyright: (C2018 Aizawa H. This is an open-access article distributed under the terms of the Creative Commons Attribution License, which permits unrestricted use, distribution, and reproduction in any medium, provided the original author and source are credited. 